\title{
A Streakline Representation of Flow in Crowded Scenes
}

\author{
Ramin Mehran ${ }^{\dagger}$, Brian E. Moore ${ }^{\ddagger}$, Mubarak Shah ${ }^{\dagger}$ \\ ${ }^{\dagger}$ Computer Vision Lab, ${ }^{\ddagger}$ Department of Mathematics \\ University of Central Florida \\ ramin@cs.ucf .edu, bmoore@math.ucf .edu, shah@eecs.ucf .edu
}

\begin{abstract}
Based on the Lagrangian framework for fluid dynamics, a streakline representation of flow is presented to solve computer vision problems involving crowd and traffic flow. Streaklines are traced in a fluid flow by injecting color material, such as smoke or dye, which is transported with the flow and used for visualization. In the context of computer vision, streaklines may be used in a similar way to transport information about a scene, and they are obtained by repeatedly initializing a fixed grid of particles at each frame, then moving both current and past particles using optical flow. Streaklines are the locus of points that connect particles which originated from the same initial position. In this paper, a streakline technique is developed to compute several important aspects of a scene, such as flow and potential functions using the Helmholtz decomposition theorem. This leads to a representation of the flow that more accurately recognizes spatial and temporal changes in the scene, compared with other commonly used flow representations. Applications of the technique to segmentation and behavior analysis provide comparison to previously employed techniques, showing that the streakline method outperforms the state-of-the-art in segmentation, and opening a new domain of application for crowd analysis based on potentials.
\end{abstract}

\section{Introduction and Related Work}

Behavior analysis in crowded scenes remains an open problem in computer vision due to the inherent complexity and vast diversity found in such scenes. One hurdle, that must be overcome, is finding good ways to identify flow patterns without tracking individual objects, which is both impractical and unnecessary in the context of dense crowds. Another hurdle is finding good ways to understand changes in behavior when the scene context and crowd dynamics can vary over such a wide range.

Several methods based on optical flow have been presented in recent years to handle these hurdles. In computer vision, optical flow is widely used to compute pixel wise instantaneous motion between consecutive frames, and numerous methods are reported to efficiently compute accurate optical flow. However, optical flow does not capture long-range temporal dependencies, since it is based on just two frames, and by itself does not represent spatial and temporal features of a flow that are useful for general applications. 
Recently, based on the Lagrangian framework of fluid dynamics, a notion of particle flow was introduced in computer vision. Particle flow is computed by moving a grid of particles with the optical flow through numerical integration, providing trajectories that relate a particles initial position to its position at a later time. Impressive results employing particle flow have been demonstrated on crowd segmentation [1] and abnormal crowd behavior detection [2]. However, in particle flow the spatial changes may be ignored, and it has significant time delays. The main goal of this paper is to introduce a notion of streaklines to computer vision with the intent to remedy these problems, and though our applications are crowd and traffic dynamics, the method of streaklines is applicable to many problems that are approached through optical flow.

Streaklines are well known in flow visualization [3,4] and fluid mechanics [5] as a tool for measurement and analysis of the flow. With regard to flow visualization, streaklines are defined as the traces of a colored material in the flow. To understand streaklines, consider a fluid flow with an ink dye injected at a particular point. If the ink is continuously injected, then a line will be traced out by the ink in the direction of the flow, this is a streakline. If the direction of flow changes, then the streaklines change accordingly.

Streaklines are new to computer vision research. In this context, streaklines may be obtained by repeatedly initializing a grid of particles and moving all particles according to the optical flow, in the spirit of a Lagrangian fluid flow. In other words, place a particle at point $p$, and move the particle one time step with the flow. In the next time step, the point $p$ is initialized with a new particle, then both particles are moved with the flow. Repeating this process on some time interval $T$ produces particle positions from which we obtain streaklines.

In video scene analysis, which is the scope of this paper, some approaches consider the entire scene as a collection of objects, and methods for scene understanding often involve object trajectory clustering and human action recognition. Examples include the tracking methods of [6] for individuals and [7] for groups of pedestrians, and the more recent work of Pellegrini et al. [8] in tracking based on social force model. Yet, the domain of application for these methods is limited to low density scenes with medium to high pixel resolutions on objects. Our work is concerned with high density scenes and low object resolution.

In other approaches, motion and tracking are represented by a set of modalities such as salient feature points [9,10], spatio-temporal volumes [11]. This promotes occlusion handling while preserving local accuracy. In the related approaches, it is common to represent both crowds and individuals as a set of regions, group of feature points, or sparse flows. In [9], Brostow and Cipolla use low level feature tracking to detect individuals in a dense crowd. Seemann et al. [12] presented a generative model to detect pedestrians as a combination of occupancy distributions.

Other methods of scene understanding involve particle tracking, motion pattern recognition, and segmentation based on dense optical flow $[13,14]$. These methods are popular due to the intrinsic ability of global approaches to handle occlusion. The framework provides insight to social/group behavior of humans 
Table 1. Advantages of Streaklines over Streamlines and Pathlines

\begin{tabular}{|l||l|l|}
\hline Streamlines & Pathlines & Streaklines \\
\hline \hline Spatial gaps in flow. & Ignores spatial changes. & Fills gaps. \\
\hline Rough transitions in time. & Time delays. & Captures instant changes. \\
\hline
\end{tabular}

in crowds, but individual tracking or action recognition is only possible through a top-down framework. Recent works of Ali and Shah [1] on crowd analysis, and $[15,16,2]$ on abnormal behavior detection fall into this category. In addition, the particle video method [17] of Sand and Teller has a potential application in crowded scenes as it was originally introduced to handle occlusions while providing dense motion information.

In this paper, we maintain three major contributions. First, we assert a streakline framework as a new tool for analysis of crowd videos. We demonstrate streaklines can be more informative than commonly used flow representations, known as optical flow and particle flow. Second, we present an innovative algorithm to compute a fluid like flow of crowds to perform behavior analysis. Third, we present potential functions as valuable tools, for behavior analysis, and compliment the streakline framework.

The capabilities of the streakline framework is tested in two applications: crowd segmentation and abnormal behavior detection. The segmentation results demonstrate an improvement for unsteady flows in comparison to state of the art. The behavior detection results show an improvement over base-line optical flow.

\section{Streaklines vs. Pathlines and Streamlines}

In fluid mechanics there are different vector field representations of the flow [5]: Streamlines are tangent to the velocity vectors at every point in the flow. These correspond to optical flow, and a visual example is given in Figure 1(a).

Pathlines are trajectories that individual particles in a fluid flow will follow. These directly correspond to integration of optical flow in time and are illustrated by a set of curves with the spectrum of colors from Blue to Orange in Figure 1(b). Particle flow is the set of pathlines which are computed from time averaged optical flow [1].

Streaklines represent the locations of all particles at a given time that passed through a particular point. Figure 1(c) shows streaklines as red curves next to pathlines.

For flows that are steady and unchanging, these three representations are the same, but for flows that are unsteady, so that directions of flow can change with time, they are different. Since we are using a Lagrangian model for fluid flow to exploit the dynamics in crowd videos, where frequent changes in the flow are expected, it is important to know which vector field representation is most appropriate for the given problem. In this work, we provide a juxtaposition of streaklines with streamlines and pathlines, which correspond to commonly used 
Table 2. A table of values for $x$-coordinate particle positions, which are computed from the optical flow. Columns correspond to pathlines and rows correspond to streaklines.

\begin{tabular}{|c|c|c|c|c|c|c|c|}
\hline & $L^{p}(0, T)$ & $L^{P}(1, T)$ & $L^{P}(2, T)$ & $\ldots$ & $L^{P}(t, T)$ & $\ldots$ & $\mathrm{L}^{\mathrm{P}}(\mathrm{T}, \mathrm{T})$ \\
\hline$S^{P}(0,0)$ & $x_{0}^{p}(0)$ & & & & & & \\
\hline$S^{P}(0,1)$ & $x_{0}^{p}(1)$ & $\mathrm{x}_{1}^{\mathrm{p}}(1)$ & & & & & \\
\hline$S^{P}(0,2)$ & $x_{0}^{p}(2)$ & $\mathrm{x}_{1}^{\mathrm{p}}(2)$ & $x_{2}^{p}(2)$ & & & & \\
\hline$S^{P}(0, t)$ & $\mathrm{x}_{0}^{\mathrm{p}}(\mathrm{t})$ & $\mathrm{x}_{1}^{\mathrm{p}}(\mathrm{t})$ & $x_{2}^{p}(t)$ & .. & $x_{t}^{p}(t)$ & & \\
\hline$S^{p}(0, T)$ & $x_{0}^{p}(T)$ & $\mathrm{x}_{1}^{\mathrm{p}}(\mathrm{T})$ & $x_{2}^{p}(T)$ & $\ldots$ & $x_{t}^{p}(T)$ & $\ldots$ & $x_{T}^{p}(T)$ \\
\hline
\end{tabular}

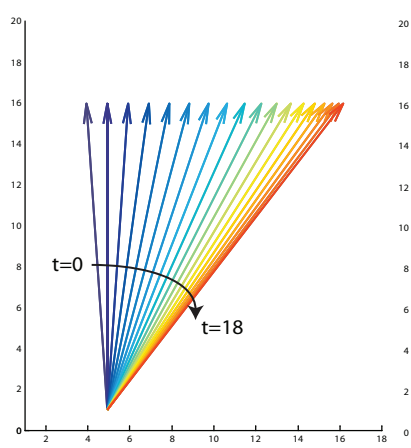

(a)

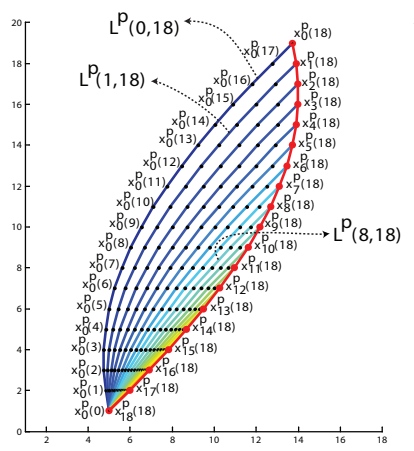

(b)

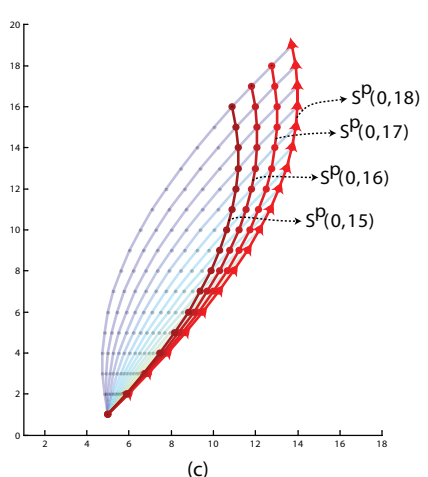

Fig. 1. An illustration of pathlines and streaklines generated using a locally uniform flow field which changes over time. (Labels on points and curves directly correspond to Table 2.) (a) The changes in the flow vectors over time period $t=0$ to $t=18$. (b) The pathlines are illustrated as a spectrum of lines. Blue corresponds to the initiating frame of $t=0$ and orange corresponds to initiating frame of $t=18$. The red line illustrates the streakline at frame $t=18$. (c) Streaklines at different frames as red curves to illustrate the evolution of the streaklines through time. The streakline at time $t=18$ is illustrated along with the initiating motion vector as explained by (2).

methods $[16,18]$ based on optical flow and particle flow, respectively. Our theory and results show that streamlines leave spatial gaps in the flow, as well as choppy transitions between frames. This is because it is produced from instantaneous velocity vectors. Hence, this approach does not produce fluid-like flow for crowd videos [19]. Pathlines overcome this problem by filling the spatial gaps, but do not allow for detection of local spatial changes, and in addition create an artificial time lag. Our streakline approach provides solutions to each of these problems, and Table 1 gives an overview of the advantages.

To explain how streaklines are computed, let $\left(x_{i}^{p}(t), y_{i}^{p}(t)\right)$ be a particle position at time $t$, initialized at point $p$ and frame $i$ for $i, t=0,1,2, \ldots, T$. Repeated initialization at $p$ implies $\left(x_{i}^{p}(i), y_{i}^{p}(i)\right)=\left(x_{0}^{p}(0), y_{0}^{p}(0)\right)$. Particle advection is achieved by

$$
\begin{aligned}
& x_{i}^{p}(t+1)=x_{i}^{p}(t)+u\left(x_{i}^{p}(t), y_{i}^{p}(t), t\right) \\
& y_{i}^{p}(t+1)=y_{i}^{p}(t)+v\left(x_{i}^{p}(t), y_{i}^{p}(t), t\right)
\end{aligned}
$$



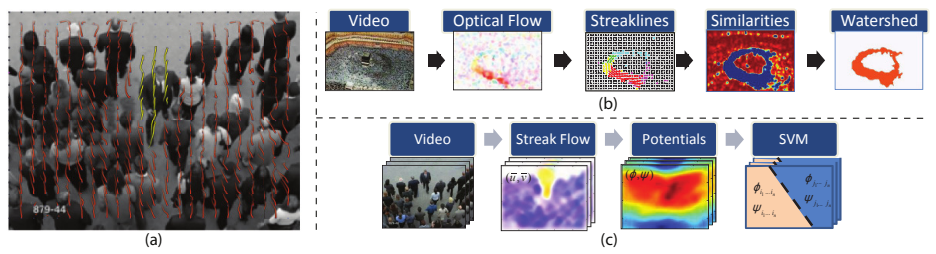

Fig. 2. (a) An illustration of streaklines for a video sequence. (b) The crowd segmentation algorithm. (c) Abnormal behavior detection algorithm.

where $u$ and $v$ represent the velocity field obtained from optical flow. This yields a family of curves, all starting at point $p$ and tracing the path of the flow from that point in frame $i$. Naturally, for steady flow all these curves lie along the same path, but for unsteady flows the curves vary in direction and shape, characteristic of pedestrian flow.

Particle advection for all $i, t=0,1,2, \ldots, T$ using (1), yields a table of values for $x_{i}^{p}(t)$ (shown in Table 2) and similarly for $y_{i}^{p}(t)$. The columns of the table show the pathlines $L^{p}(t, T)$, which are the particle trajectories from time $t$ to $T$. The rows provide the streaklines $S^{p}(0, t)$, connecting all particles from $t$ frames that originated at point $p$. Corresponding to this table, Figure 1 illustrates the set of streaklines and pathlines for an example unsteady flow at time $t=T$. At the start of observation, particles are initiated at every time instant at point $p$. The spectrum of lines from blue to orange represents the pathlines of particles which have been initiated at time $t=0$. The solid red color lines depict streaklines. Since the flow is not steady, the streaklines and pathlines are different.

The unsteady flow at a point can be represented by either a set of pathlines or a streakline. However, the streakline provides a speed and memory gain, as a streakline with $L$ particles corresponds to $L$ pathlines with $L \times(L-1) / 2$ particles. There are other interesting, less obvious, properties that streaklines inherit from fluid mechanics. First, in unsteady flows, extra long streaklines may exhibit shapes inconsistent with the actual flow, meaning they can not be allowed to get too long [20]. Second, as invented for visualization purposes, streaklines in fluids transport a color material along the flow, meaning they propagate changes in the flow along their path. Similarly, our setup allows streaklines to propagate velocities, given by the instantaneous optical flow $\Omega=(u, v)^{T}$ at the time of initialization, along the flow like a material. To this end, we define an extended particle $i$ as a set of position and initial velocity

$$
P_{i}=\left\{x_{i}(t), y_{i}(t), u_{i}, v_{i}\right\}
$$

where $u_{i}=u\left(x_{i}^{p}(i), y_{i}^{p}(i), i\right)$, and $v_{i}=v\left(x_{i}^{p}(i), y_{i}^{p}(i), i\right)$. In the whole scene, we consider only streaklines comprising extended particles. Figure 2.a depicts streaklines for an example sequence. 


\section{Computations with Streaklines}

Streaklines provide a means to recognize spatial and temporal changes in the flow, that neither streamlines nor pathlines could provide directly. This point is made here using streak flow and potential functions. In essence, streak flow is obtained by time integration of the velocity field, while potential functions are obtained from spatial integration, and each provides useful information concerning the dynamics in the scene.

\subsection{Streak Flow}

Research in social behavior of pedestrians in crowds reveals that people tend to follow a pathway trailing pedestrians who have similar paths as a group [21]. As a pedestrian passes a point, there is a social expectation that any other pedestrian behind him/her would follow a similar path. Considering this social behavior, the actual, but invisible, flow of pedestrians has no gaps between individuals who are walking similarly. Hence, for crowd motion, gaps in the optical flow should be filled along trajectories with similar motion vectors prior to analysis.

In order to achieve an accurate representation of flow from crowd motion, we use the streaklines to compute a new motion field which we refer to as streak flow, denoted $\Omega_{s}=\left(u_{s}, v_{s}\right)^{T}$. To compute streak flow, we compute the streaklines by temporally integrating optical flow, as illustrated in Table 2, and forming the particles as in Equation (2). We describe the computation of $u_{s}$; computation of $v_{s}$ is similar. Given data in the vector

$U=\left[u_{i}\right]$, where $u_{i} \in P_{i}, \forall i, p$, we compute the streak flow in the $x$ direction at each pixel.

Based on equations (1), particle positions have sub-pixel accuracy. We compute a triangulation of pixels, which implies that each particle $P_{i}$ has three neighboring pixels (nearest neighbors). At the sub-pixel level, it is reasonable to consider $u_{i}$ to be the linear interpolation of the three neighboring pixels. Hence, we define

$$
u_{i}=a_{1} u_{s}\left(k_{1}\right)+a_{2} u_{s}\left(k_{2}\right)+a_{3} u_{s}\left(k_{3}\right),
$$

where $k_{j}$ is the index of a neighboring pixel, and $a_{j}$ is the known basis function of the triangulation of the domain for the $j$-th neighboring pixel. Using a triangular interpolation formula, each $u_{s}\left(k_{i}\right)$ is computed based on the relative positions of the three pixels and the particle. Using (3) for all the data points in $U$, we form a linear system of equations

$$
A u_{s}=U,
$$

where $a_{i}$ are entries of the matrix $A$, and $u_{s}$ is the least square solution of (4). ${ }^{1}$

Streak flows encapsulate motion information of the flow for a period of time. This resembles the notion of particle flow (equivalent to average optical flow) where advection of a grid of particles over a window of time provides information for segmenting the crowd motion. We argue that streak flows exhibit changes in

\footnotetext{
${ }^{1}$ www.mathworks.com/matlabcentral/fileexchange/8998-surface-fitting-using-gridfit
} 


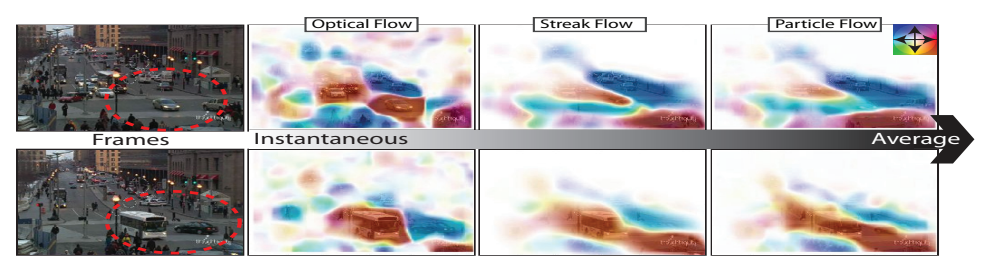

Fig. 3. The comparison of optical flow, particle flow and streak flow for Boston sequence (color coded). The red circle indicates the area to notice.

the flow faster than particle flow, and therefore, they capture crowd motions better in a dynamically changing flow. This can be observed in Figure 3, illustrating sample frames from a video of a traffic intersection, which includes motions from both pedestrians and vehicles. The flow in the scene is unsteady and the different motion patterns appear in the video as the traffic lights change. The figure compares the streak flow to the particle flow and the optical flow in capturing temporal and local changes. For temporal changes the flow is compared at two different times: (1) A the start of the top-down flow of traffic (1st row), and (2) at the ending stage of the up-down traffic flow (2nd row).

Temporal changes: The first row of Figure 3 shows a frame from the sequence a few seconds after the change of a traffic light, so vehicles and pedestrians are now moving in a different direction, from top to bottom. By comparing the area to notice inside the red circle, it is evident that the streak flow is able to capture this change after only a couple of frames, but the particle flow lags in shaping to the new flow, and the optical flow shows choppy flow segments that are difficult to use for further analysis.

Local changes: Both streak flow and particle flow have the ability to fill in the gaps of the non-dense traffic flow. In second row of Figure 3, the optical flow shows the motion of a car making a left turn. The particle flow is unable to capture this change, and the region on the bus and car both show inconsistency compared to instantaneous flow. The figure shows that the streak flow was more accurate in exhibiting immediate flow changes over the car as well as the bus.

\subsection{Potential Functions}

Building on the fluid dynamics approach to crowd motion, we employ another concept from fluids providing a different point of view. In simplified mathematical models of fluids, it is often assumed that the fluid is imcompressible, and irrotational. These assumptions imply several conservation properties of the fluid, but most importantly, they lead to potential functions, which are scalar functions that characterize the flow in a unique way. For this discourse, potential functions enable accurate classification of behaviors in a scene, which is not possible with streak flow alone. Application of potential functions to abnormal behavior detection is presented in Sections 4 and 5 . 
Since the optical flow $\Omega=(u, v)^{T}$ denotes a planar vector field, the Helmholtz decomposition theorem states that $\Omega=\Omega_{c}+\Omega_{r}$, where $\Omega_{c}$ and $\Omega_{r}$ respectively denote the incompressible and irrotational parts of the vector field. To clarify, an incompressible vector field is divergence free $\nabla \cdot \Omega=0$, and an irrotational vector field is curl free $\nabla \times \Omega=0$. Thus, there are functions $\psi$ and $\phi$, known respectively as the stream function and the velocity potential, satisfying $\Omega_{c}^{\perp}=\nabla \psi$ and $\Omega_{r}=\nabla \phi$ (see, for example [5]). Following [22], we use Fourier transforms to decompose incompressible and irrotational parts of the vector field and estimate the potential functions using

$$
\begin{aligned}
& \phi(x, y)=\phi_{0}+\frac{1}{2} \int_{0}^{x}\left(u_{r}(s, y)+u_{r}(s, 0)\right) d s+\frac{1}{2} \int_{0}^{y}\left(v_{r}(x, s)+v_{r}(0, s)\right) d s \\
& \psi(x, y)=\psi_{0}+\frac{1}{2} \int_{0}^{y}\left(u_{c}(x, s)+u_{c}(0, s)\right) d s-\frac{1}{2} \int_{0}^{x}\left(v_{c}(s, y)+v_{c}(s, 0)\right) d s
\end{aligned}
$$

Potential functions are computed in Corpetti et al. [22] and used in a meteorological application to track weather patterns in satellite images. In order to compute valid potential fields, one needs a dense motion field. In that particular application the motion fields are as dense as possible, but in crowd videos the degree of motion density can vary by large amounts. In addition, a potential function computed directly from optical flow is noisy with many valleys and peaks, which quickly disappear and reappear. Streak flows enable us to compute reliable potential functions for crowd flow, incorporating local and temporal changes. In other words, we incorporate streaklines to compute smoothly evolving potential functions, which better reveal the dynamics of the crowd. In a broad view, the stream function $\psi$ provides the information regarding the steady and nondivergent part of the flow, whereas the velocity potential $\phi$ contains information regarding the local changes in the non-curling motions. Moreover, to have a complete picture of the flow we need information from both potential functions. With this perspective, we illustrate the strength of potentials in discriminating lanes and divergent/convergent regions in five different scenes in Figure 4. In this figure, the velocity potential is accountable for capturing unsteady changes in the flow. For instance, escape to the sides of the scene corresponds to a valley in the center of $\phi$ and formation of surrounding peaks on the sides. Furthermore, the stream function $\psi$ is incorporated to detect lanes in the steady motion of vehicles. The area between contours of $\psi$ (i.e. streamlines) show the regions of steady and non-divergent motion such as lanes. The algorithm for detection of lane and divergent/convergent regions is explained in Section 4.

\section{Applications of Streaklines}

Using streak flow and potential functions, we demonstrate the strength of our approach for crowd segmentation and abnormal behavior detection in unsteady flows. In the end, we find that our method performs better than other methods for solving these problems. 


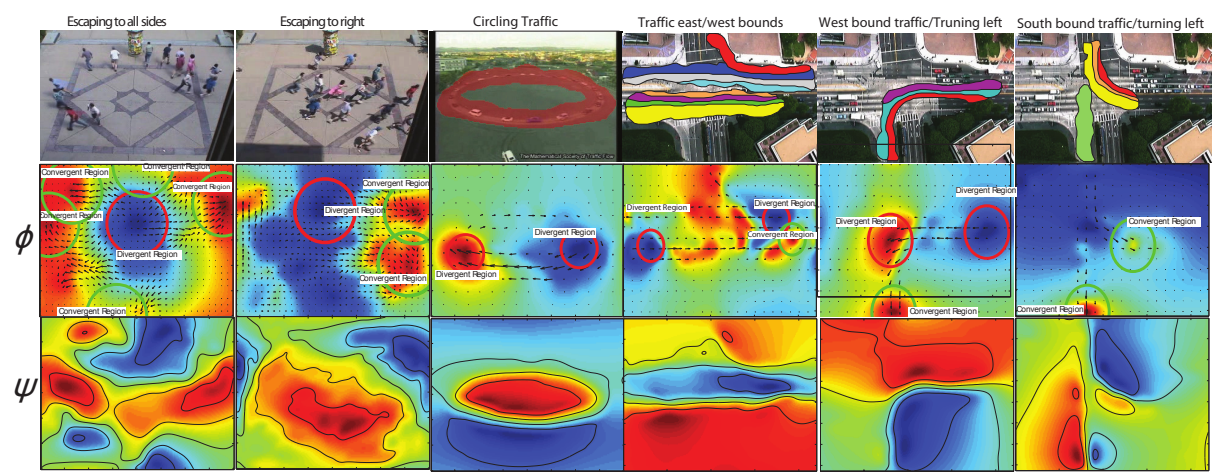

Fig. 4. An illustration of discrimination power of potentials for six manually labelled behaviors. The first two columns, escape panic from UMN Dataset [23], column 3 shows circulating motion of cars in a lane, and column 4 to 6 show traffic forming lanes from NGSIM dataset. Potentials are scaled to maximum value and plotted using jet colormap. (1st row) The lanes are overlaid the frame for the steady motions. (2nd row) divergent regions (red circles) and convergent regions (green circle). (3rd row) Streamlines, which are contours of stream function.

\subsection{Crowd Segmentation}

In this algorithm, we segment every frame of the video into regions of different motions based on the similarity of the neighboring streaklines. Similar streaklines correspond to similar trajectories of particles passing from neighboring pixels over a period of time. Hence, it captures the affinity of current and previous motions at these pixels. Figure 2.b presents the block diagram of the segmentation algorithm. First, frame by frame optical flow of the video is computed. Using the optical flow, a set of particles are then moved over the frame to construct the streaklines and the streak flow. These quantities are used to compute similarity in a 8-connectivity neighborhood. For every pair of pixels $i$ and $j$, the similarity is computed in terms of streaklines and streak flow.

Each pixel is associated with a streakline of length $l$. The streakline similarity is computed using the sum of the normalized projections of internal vectors as $R_{s}(i, j)=\sum_{m=0}^{l-1} \operatorname{prj}\left(X_{m}^{i}, X_{m}^{j}\right)$, where $X_{m}^{i}$ and $\operatorname{prj}(\cdot, \cdot)$ are defined in Figure 5.a. Streak flow similarity is computed as $R_{\Omega}(i, j)=\left|\cos \left(\angle \Omega_{s}^{i}\right)-\cos \left(\angle \Omega_{s}^{j}\right)\right|$, where $\angle \Omega_{s}^{i}$ is the angle of the streak flow vector at pixel $i$. In order to define boundaries of the regions, we compute the similarity map at every pixel using

$$
H(i)=\sum_{j \in N(i)} \alpha R_{s}(i, j)+\beta R_{\Omega}(i, j),
$$

where $\alpha$ and $\beta$ are weights regulating the share of streakline and streak flow similarities in the final segmentation. We use $\alpha=0.8$ and $\beta=0.2$ in the experiments. Since similar motions over time build similar streaklines and streak flows, boundaries of different motions form valleys in the similarity map. Using 

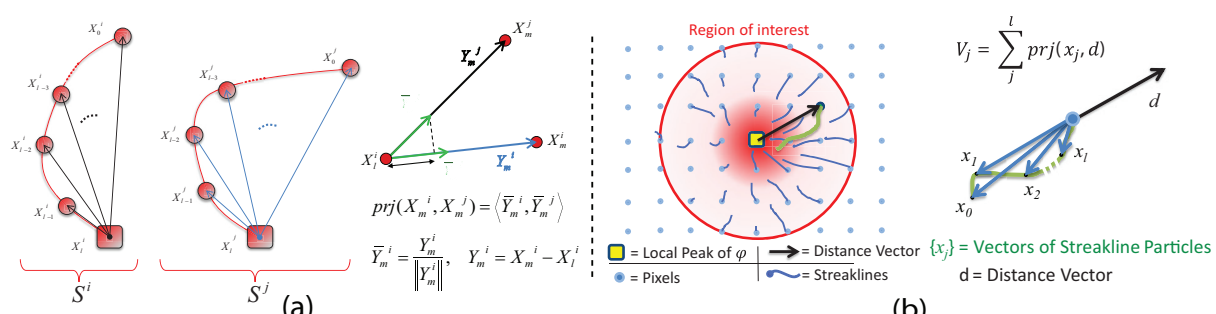

(b)

Fig. 5. (a) Streaklines $S^{i}$ and $S^{j}$ are sets of vectors $X_{1 . . L}^{i}$ and $X_{1 . . L}^{j}$. The originating point of streaklines (rectangle), the particles (circles), and the normalized projections of the vectors are used for computing the similarity of streaklines. (b) The computation of divergence factor, $V_{i}$, for a region of interest.

the negative of the similarity map, we segment the crowd into regions of similar motion with watershed segmentation. Results are presented in Section 5.1.

Lane detection: In addition to segmenting a frame into regions of consistent motion, we combine information from potentials to detect lanes in each segment. As stated in section 3.2, the area between contours of $\psi$ corresponds to the steady flow, and the rate of the incompressible flow between a pair of contours is equal to the difference between the values of $\psi$ on those contours. Considering this, we detect lanes as parts of a segmented region that fall between two contours of the stream function by a simple intersection operation (see Figure 4).

\subsection{Abnormal Behavior Detection}

To detect abnormal behavior of crowds, it is necessary to have a global picture of the behavior in a scene, for which we use potential fields. The surfaces $\phi$ and $\psi$ characterize particle positions and velocities in a global sense, and abnormal behaviors are simply detected as large deviations from the expected. Here, we present an algorithm to detect abnormal behavior in crowds using potential functions for the flow.

Figure 2.c shows the block diagram for the algorithm. For every frame in a video sequence, the Streak flow $\Omega_{s}=\left(u_{s}, v_{s}\right)^{T}$ is computed, and the potential functions of the frame $\{\phi, \psi\}$ are computed using equations (5) and (6). The peaks and valleys of the potential surface convey information regarding the global behavior of the flow (Figure 4). Thus, potentials provide new features to distinguish global behaviors in the crowd in compact form. For every frame, a feature vector $V$ is formed by concatenating the values of $\phi$ and $\psi$ of that frame. Using feature vector $V$, we recognize behaviors in each frame by training a support vector machine (SVM) classifier. In Section 5 , we provide comparative results of abnormal behavior detection using potentials.

In addition to detecting abnormal behaviors, we incorporate streaklines and the velocity potential $\phi$ to provide a description of the anomaly based on divergent/convergent regions. The extrema on velocity potentials correspond to divergent or convergent regions. To robustly detect these regions, we find the 
major local extrema of $\phi$, and then compute the average divergence factor, $\bar{V}=\frac{1}{n} \sum_{i} V_{i}$, where $V_{i}$ is defined in Figure 5.b, and $n$ is the number of pixels in the radius $r$ of the extremum point. Simple thresholding of this factor distinguish divergent/convergent regions as Region Type $=\left\{\begin{array}{ll}\text { Divergent, } & \text { if } \bar{V}>T \\ \text { Convergent, } & \text { if } \bar{V}<T\end{array}\right.$. In the experiments, $r$ is set fixed empirically for each scene and $T=0$. As it is illustrated in Figure 4 the escape panic scene involves the divergent region in the center and convergent regions on the sides to which the crowd is running. Similarly, a sudden change in the direction of turning vehicles or the entry/exit points form divergent/convergent regions. The circular regions in the second row are the actual output of our algorithm. Obviously, there are some mistakes $(20 \%)$. For example, in circling traffic, column 3, the region on the right is detected incorrectly.

\section{$5 \quad$ Experimental Results}

We present results of algorithms outlined in Section 4, using experiments on two datasets. A stock footage dataset from the web [2] is used for streakline analysis, and a dataset from the University of Minnesota [23], which contains 11 videos of crowd escape panic, is used to evaluate the effectiveness of potentials for abnormal behavior detection.

\subsection{Results of Crowd Motion Segmentation}

Results of our proposed segmentation algorithm are provided here. We compare with the state of the art [1], considering crowds with dynamic segmentations, such that the motion patterns vary in time exhibiting different states of behavior.

Figure 6 provides segmentation results for two scenes, and video frames are overlaid by colored segmentation regions. In this experiment, the length of streaklines and pathlines is $l=40$. On the left side of Figure 6 , an intersection is shown in Boston, containing three behavioral phases represented by frames 40 , 197, and 850. (1) South bound traffic is formed. (2) Traffic lights change and an east/west bound (from/to station) a flow of pedestrians emerges. (3) Traffic lights change again, and a north bound vehicle flow is formed together with an east bound pedestrian flow. On the right side of Figure 6, an intersection is shown in Argentina containing three behavioral phases. (1) East/west bound traffic is formed. (2) After the traffic lights change, a south bound vehicle flow and a north/south pedestrian flow develop. (3) Traffic lights change to the first phase and east/west bound flows resume. Frames 115 and 213 illustrate the start of phases 2 and 3, respectively. The optical flow of this video is particulary noisy as it is based on time-lapse imagery, whereas the Boston sequence is a regular $30 \mathrm{fps}$ video. Videos are available in the supplementary material.

Figure 6 demonstrates segmentations based on streaklines are spatially and temporally pronounced and more accurate in dynamic scenes than the state of the art. We highlight the gains in using our method in each frame: (Frame 40) A 

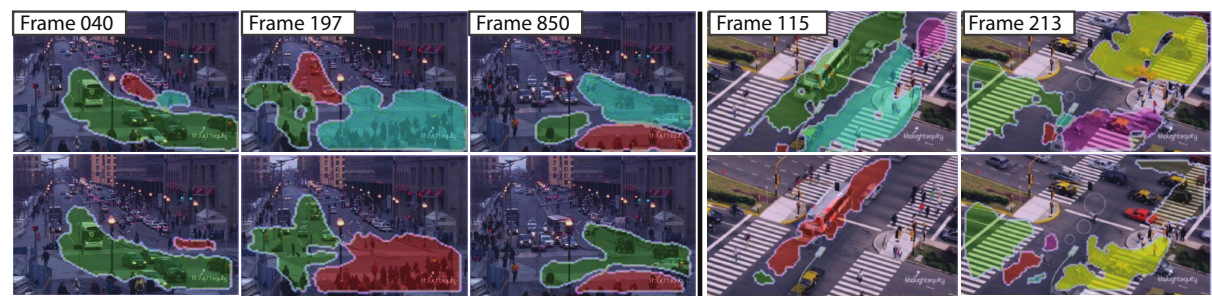

Fig. 6. Comparison of segmentation results using streaklines (1st row), and pathlines [1] (2nd row) for scenes with unsteady motions.

walking pedestrian and the north bound vehicle motion are segmented correctly. (Frame 197) Pedestrians are distinguished from the south bound cars. (Frame 850) A south bound pedestrian (first row, green) is separated from north bound vehicles. (Frame 115, 4th column) Different pedestrian flows are distinguished (first row, cyan and purple). (Frame 213) West bound vehicle flow (first row, yellow) is segmented earlier, at start of phase 2 of the video.

In Figure 7, the quantitative comparison of the proposed segmentations method and [1] is provided. In this experiment, frame by frame segmentations of both methods are compared as following. The number of objects (human/vehicle) in the each segmented region is counted provided that its direction of motion is no more that 90 degrees apart from the direction motion of the majority of the objects. We refer to this number as the number of correctly segmented objects (see Figure 7.a). To evaluate the methods, this number is counted manually for a subset of frames of Boston and Argentina video sequences. Figure 7 demonstrates that streakline segmentation outperforms the state of the art in number of correctly and incorrectly segmented objects.

\subsection{Results of Abnormal Behavior Detection}

This section illustrates results for abnormal behavior detection on the UMN dataset [23], containing 11 sequences for 3 scenes. In this dataset, pedestrians initially walk randomly, and exhibit escape panic by running in different directions in the end. Figure 4 shows that potential functions provide rich information about global behavior. Interesting properties of potentials are revealed as we compare $\phi$ for frames where people escape to all sides to the frames which people run in a single direction (2nd column).

In order to illustrate the strength of potentials in representing the global behavior we compared our method using different features. In experiment (a), we first use frame-based potentials as the input features $V$ for training a SVM with RBF kernels. Second, we use vectorized streak flow $\Omega_{s}=\left(u_{s}, v_{s}\right)$ and third, we use average baseline optical flow (pyramidal LK) to perform the same task. Figure 7.e compares the recognition results using any of these three features for a different number of training examples. In order to reduce the computation time, we downsample the features of each frame by factor of $n=20$. In this experiment, 

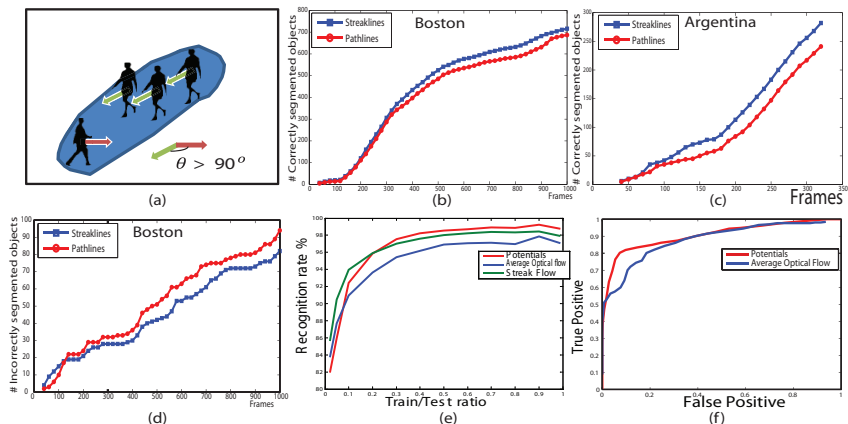

Fig. 7. (a) The criterion for segmentation evaluation, (green) correctly segmented object, (red) incorrectly segmented object. (b), (c), and (d) Quantitative comparison of segmentation results using streaklines (blue), and pathlines [1] (red). (e,f) Abnormal behavior recognition, (e) Variation of the number of training examples. (f) ROC of the cross validation.

the frames from different scenes in the dataset are combined in a single pool and a portion is selected as the train set and the rest is considered as the test set (no overlaps). The figure shows that after increasing the number of examples to merely $20 \%$, the potentials show considerable improvement in performance. In addition, the figure illustrates the strength of streak flows compared to particle flow in providing information for abnormal behavior detection.

In experiment (b), we performed a leave-one-out cross validation on the UMN dataset using downsampled version of potentials and average optical flow. In this experiment, we trained a SVM with RBF kernels on 10 videos and computed the false positive and true positives on one video sequence and repeating this for all the 11 videos. Figure 7.f illustrates the ROC of this experiment which indicates improvement using potentials over baseline optical flow.

\section{Conclusion}

Based on a Lagrangian particle dynamics framework for fluid flow, we juxtapose three vector field representations of the flow, given by streamlines, pathlines and streaklines. With application to problems in segmentation and abnormal behavior detection for crowd and traffic dynamics, we show that the streakline representation is advantageous. When compared to the other two representations, which are commonly used to solve problems in computer vision, streaklines demonstrated the ability to quickly recognize temporal changes in a sequence, in addition to finding a balance between recognition of local spatial changes and filling spatial gaps in the flow. When used to compute potential functions and to perform segmentation, the streakline approach was superior to using optical flow and comparable to using particle flow, aside from the ability to recognize scene changes. With regard to abnormal behavior detection, the method of streaklines 
proved superior to both of the other representations, and the introduction of potential functions for this purpose proved valuable.

Acknowledgments: This research was supported by the U.S. Army Research Laboratory and the U.S. Army Research Office under grant number W911NF09-1-0255.

\section{References}

1. Ali, S., Shah, M.: A lagrangian particle dynamics approach for crowd flow segmentation and stability analysis. (2007) CVPR.

2. Mehran, R., Oyama, A., Shah, M.: Abnormal behavior detection using social force model. (2009) CVPR.

3. Wijk, V., Jarke, J.: Image based flow visualization. In: SIGGRAPH. (2002)

4. Helman, J., Hesselink, L.: Visualizing vector field topology in fluid flows. IEEE Comput. Graph. Appl. 11 (1991) 36-46

5. Landau, L., Lifshitz, E. Advanced Mechanics of Fluids (1959)

6. Johnson, N., Hogg, D.: Learning the distribution of object trajectories for event recognition. (1995)

7. Marques, J.S., Jorge, P.M., Abrantes, A.J., Lemos, J.M.: Tracking groups of pedestrians in video sequences. In: Proc. CVPRW. (2003)

8. Pellegrini, S., Ess, A., Schindler, K., van Gool, L.: You'll never walk alone: Modeling social behavior for multi-target tracking. In: ICCV. (2009)

9. Brostow, G., Cipolla, R.: Unsupervised bayesian detection of independent motion in crowds. In: CVPR. (2006)

10. C. Garate, P.B., Bremond, F.: Crowd event recognition using hog tracker. (2009)

11. Ke, Y., Sukthankar, R., Hebert, M.: Event detection in crowded videos. In: ICCV. (2007)

12. Seemann, E., Fritz, M., Schiele, B.: Towards robust pedestrian detection in crowded image sequences. In: CVPR. (2007)

13. Ali, S., Shah, M.: Floor fields for tracking in high density crowd scenes. In: ECCV, Berlin, Heidelberg, Springer-Verlag (2008) 1-14

14. I. Saleemi, L. Hartung, M.S.: Scene understanding by statistical modeling of motion patterns. In: CVPR. (2010)

15. Kratz, L., Nishino, K.: Anomaly detection in extremely crowded scenes using spatio-temporal motion pattern models. In: CVPR. (2009)

16. Andrade, E.L., Blunsden, S., Fisher, R.B.: Modelling crowd scenes for event detection. In: ICPR. (2006)

17. Sand, P., Teller, S.: Particle video: Long-range motion estimation using point trajectories. In: CVPR. (2006)

18. Courty, N., Corpetti, T.: Crowd motion capture. Comput. Animat. Virtual Worlds 18 (2007) 361-370

19. Hughes, R.: The flow of human crowds. Annual Review of Fluid Mechanics 35 (2003) 169-182

20. Hama, F.R.: Streaklines in a perturbed shear flow. Phys. Fluids 5 (1962) 644-650

21. Helbing, D., Molnar, P.: Social force model for pedestrian dynamics. Physical Review E 51 (1995)

22. Corpetti, T., Memin, E., Perez, P.: Extraction of singular points from dense motion fields: An analytic approach. Journal of Mathematical Imaging and Vision (2003)

23. University of Minnesota - Crowd Activity Dataset, http://mha.cs.umn.edu/Movies/Crowd-Activity-All.avi. 